\title{
Carnets
}

Revue électronique d'études françaises de l'APEF

Deuxième série - 1 | 2014

L'étranger

\section{L'« histoire véritable de la planète Mars »}

Pour une morphologie de l'étranger et du voyage interplanétaire

\section{Maria Luísa Malato Borralho}

\section{(2) OpenEdition}

Journals

Édition électronique

URL : http://journals.openedition.org/carnets/1230

DOI : $10.4000 /$ carnets. 1230

ISSN : 1646-7698

Éditeur

APEF

\section{Référence électronique}

Maria Luísa Malato Borralho, «L'« histoire véritable de la planète Mars » », Carnets [En ligne], Deuxième série - 1 | 2014, mis en ligne le 30 mai 2014, consulté le 20 avril 2019. URL : http://

journals.openedition.org/carnets/1230; DOI : 10.4000/carnets.1230

Ce document a été généré automatiquement le 20 avril 2019.

\section{(c) (i) \&}

Carnets est mis à disposition selon les termes de la licence Creative Commons - Atribution - Pas d'utilisation commerciale 4.0 International. 


\title{
L'« histoire véritable de la planète Mars »
}

\author{
Pour une morphologie de l'étranger et du voyage interplanétaire
}

Maria Luísa Malato Borralho

1 Nous avons signalé en 2008, dans un catalogue de livres anciens, un ouvrage qui avait échappé à nos recherches sur les utopies dans les bibliothèques publiques portugaises. Les renseignements obtenus ne confirmaient que sa rareté : le texte, - en prose -, se présentait comme une traduction, fidèle, en portugais, d'un livre français, d'Henri Montgolfier, né à Marseille, peu avant la Révolution française. Publié à Lisbonne, en 1921, plus de cent ans après, le livre nous attirait surtout par son titre: História Autêntica do Planeta Marte (L'Histoire Véritable de la Planète Mars). L'entrée de la base de données Porbase, se référant à cette traduction (exemplaire de la Bibliothèque Nacionale, L 16323v), ne possédait aucune information sur l'auteur étranger. Elle nous indiquait seulement l'existence de plusieurs ouvrages scientifiques de son traducteur portugais, José Nunes da Matta (1846-1945), notamment une deuxième édition sur la description et l'usage des instruments de navigation maritime (Descripção e uso dos instrumentos de navegação..., Lisboa, Férin, 1909), la discussion d'une thèse de Laplace sur la vie dans l'univers (A Vida do Cosmos Infinit..., Lisboa, Imprensa Nacional, 1915) et un Mémoire descriptif, écrit en français, sur "un nouveau type d'avion de terre et aussi de mer» (Lisboa, Libanio da Silva, 1928).

2 Peut-être pour tout cela, la dimension scientifique de l'œuvre sur la planète Mars étaitelle prévisible. Mais la vraisemblance résistait mal à un deuxième regard. Le soupçon commençait déjà par le silence généralisé sur l'auteur, Henri Montgolfier, dans les études sur les utopies ou les catalogues des principales bibliothèques publiques françaises. Son nom de famille, celui des inventeurs des ballons aérostatiques, à air chaud, étant associé aux « montgolfières » depuis les premiers essais en 1782, faisait croire à un intérêt d'un des frères Montgolfier pour les questions d'astronomie et la planète Mars. Mais la recherche bibliographique nous rappelait vite pourtant que les frères s'appelaient JosephMichel et Jacques-Etienne, Henri étant un membre complètement inconnu. 
Par contre, le profil du traducteur devenait de plus en plus réel. Le nom de José Nunes da Matta, militaire de la Marine portugaise, directeur prestigieux de l'École Navale de Lisbonne ( $c f$. Lima, 2010), renvoyait le lecteur à un mémoire historique intéressant sur l'incendie de l'École Navale en 1916, qui a détruit une grande partie des documents de l'Arsenal et de l'observatoire de la Marine (Problemas de navegação e notas referentes ao incêndio da Escola Naval, Lisboa, Imprensa Nacional, 1916). Mais il était surtout associé à l'adhésion du Portugal à l'Heure Internationale, et à l'adoption du méridien de Greenwich comme méridien zéro. Question passionnante, que les lecteurs de Le tour du monde en 80 jours de Jules Verne avaient déjà suivie attentivement en 1872, en feuilletons, mais décision politiquement très contestée à l'époque, aussi bien au Portugal qu'ailleurs, puisque l'heure de Greenwich signifiait la norme d'Angleterre. Sur le sujet, et pour faciliter la compréhension du Décret du 26 mai 1911 promulgué par la République Portugaise, José Nunes da Mata a même publié, en 1912, A nova hora e os fusos horários.

4 Avant 1910, la Monarchie avait été accusée de se plier à l'influence anglaise. Mais faire marcher le Portugal républicain selon l'Heure Internationale («anglaise ») était une question simultanément pragmatique et symbolique. Et José Nunes da Mata, pesant les deux sortes d'arguments (le scientifique et le politique), démontrait, - ce nous semble -, un indéniable profil utopique auquel les chercheurs d'utopies commencent à se montrer sensibles. D'après sa bibliographie, il avait manifesté sa vie durant un enthousiasme constant pour les causes publiques, pour les grandes questions sur la construction de l'humanité : parmi les articles scientifiques, les discussions académiques et les articles sur les projets maritimes, on reconnaît certains mots-clés de la bibliographie d'un utopiste : « l'avenir » « l'Humanité », « la paix universelle », la « constitution », le « décalogue » des «droits et des devoirs » des citoyens, la « Raison », la «Justice » et le « rêve », une envie de « pureté »: Projecto de Constituição (1911), Divagações em verso : Paz e Esterilização (s.d.), 0 Decálogo de Jefferson (1926), Miseranda Humanidade (1939), A Sabedoria Humana ou Dezasseis Deveres dos Cidadãos (tous deux de 1942), etc...

5 Si on part de la pensée d'A. Neussus et de l'idée selon laquelle l'utopie est un tableau de l'avenir fondé sur la négation critique du présent, où l'auteur lutte contre les contrevaleurs de l'injustice et du malheur (Neussus, 1980 : passim), il faut commencer par reconnaitre ici cette tension entre deux temps et deux espaces: l'avenir et un espace inconnu et plus beau vs. le présent et l'espace réel, trop imparfait. Bien significatif s'avère le titre et le texte de José Nunes da Matta, Caos ou o Pandemónio humano e meio de o evitar (Lisboa, Imp. Beleza, 1931). L'utopiste est un déclencheur de nouveaux points de vue. L'utopiste, tout comme l'étranger, change de perspective en se déplaçant. C'est à travers le voyage qu'il déplace notre regard commun, qu'il interroge nos lieux-communs. Mais il n'est jamais étranger aux choses humaines. Selon Raymond Trousson, le vrai utopiste serait même le contraire du misanthrope : il est généralement un juriste, un médecin, un politique, quelqu'un qui souhaite soigner les plaies du corps social, qui ne fuit jamais ses responsabilités, qui en est même passionné :

Cette tendance fondamentale de l'utopiste à refuser l'action concrète au profit de la spéculation conduit trop vite à conclure qu'il verse dans la chimère. S'agit-il bien d'un rêve pur, d'un déni radical de toute réalité ? L'examen révèle qu'il n'y a que rarement rupture avec le réel, mais correction, rectification de ce réel. L'utopiste, constatant ce qui est, imagine et suggère ce qui devait être : il est obsédé par un « devoir-être ». (Trousson, $1979: 17$ ).

6 Tout compte fait, il faut bien se méfier des indications de la couverture. L'auteur est le traducteur et le nom de l'auteur, Montgolfier, ne constitue qu'une stratégie de 
dissimulation, ou plutôt de simulation. Comme on le constate fréquemment dans les récits utopiques, le véritable auteur se dissimule en tant que récepteur (narrataire et/ou traducteur et/ou éditeur...) d'un texte d'autrui. Ou plutôt, il simule les oppositions entre les narrateurs et les narrataires en inversant parfois leurs fonctions. L'utopie vit de ce dialogue forcé, de cette responsabilité dispersée qui la rend dynamique et " adogmatique ». Cette simulation diminue, d'ailleurs, le risque de parler à contrecourant.

7 L'Utopie de Thomas More, texte fondateur du paradigme, en est un exemple. More en est le narrataire, et Hytlodé, le narrateur principal, est un étranger, un Portugais qui a beaucoup voyagé et qui est le seul connaisseur de l'efficace du gouvernement de l'île de l'Utopie. Soulignons en outre que la figure de l'étranger y joue un rôle ironique (Jankélevitch, 1979 : passim). La crédibilité de l'étranger est, bien plus que celle du fou ou de l'enfant, une crédibilité qui s'apparente à celle de l'acteur ou de l'histrion, puisque sa condition est celle du masque, et sa force, ironique, s'appuie sur la faiblesse de son point de vue. Le nom «étranger » (bien plus que le fou ou l'enfant, voire que l'acteur) est étymologiquement une description de déplacement vers un extérieur (« ex-strare ») : si le voyageur peut ne pas être un voyageur, l'étranger, lui, est toujours un voyageur, par hasard ou par nécessité, volontairement ou involontairement. D'ailleurs, qui est « l'étranger »? Toujours une perspective, et rien d'autre : un voyageur qui se déplace vers un lieu qu'il commence par désigner «l'étranger». Et pourtant, quand il y arrive, il devient lui-même «l'étranger » pour ceux qui le regardent alors...

8 En ouvrant l'ouvrage "d'Henri Montgolfier», les jeux de simulation commencent précisément par ces variations du point de vue, entre un Français du XviII ${ }^{\mathrm{e}}$ siècle (Henri de Montgolfier, habitant de la planète Mars, voyageur français, né à Marseille, le 25 janvier 1775, à la veille de la Révolution française) et un Portugais du $\mathrm{xx}^{\mathrm{e}}$ siècle (José Nunes da Matta, Portugais, habitant de Guernesey, traducteur, éditeur, protagoniste de la $1^{\text {ère }}$ République portugaise). Qui est l'étranger, ici? L'auteur français, le premier propriétaire du texte, qui habite Mars, ou le traducteur portugais, son héritier provisoire, narrateur qui habite l'île de Guernesey ? La note de présentation du traducteur commente les références de la couverture. En fait, elle les confirme et les nie simultanément. La traduction est ici véritablement l'espace où les formes des mots traînent les sens des mots ("traduire» vient de "trahere", trainer) : entre le manuscrit et le livre, la forme se déplace, soit pour retenir, soit pour perdre une signification centrale.

En effet, «l'auteur » et « le traducteur » sont tout d'abord liés par cette traction et par cette trahison. Frères dans le malheur du présent et frères dans le bonheur de l'avenir, ils ne sont que les véhicules d'une description, d'une idée qu'ils baladent entre quatre lieuxlimites: Mars (planète " habitée ", où l'auteur écrit); Guernesey (l'île où le traducteur trouve le texte); Parede (aux alentours de Lisbonne, où le traducteur signe la traduction) et Paris (ville jamais rejointe où le manuscrit devait rester, dans un musée). Le jeu se maintient même dans les livres offerts par José Nunes da Matta à ses amis. L'exemplaire du livre História Autêntica do Planeta Marte que nous avons acheté, et qui a appartenu à la famille d'António Lopes de Oliveira, possède même une dédicace amusante de l'auteur, José Nunes da Matta. Il prolonge le jeu: «Ao seu estudioso amigo e distinto aluno do Liceu, Snr. António Ba. ${ }^{2}$ Lopes de Oliveira, em nome do autor, oferece o tradutor José Nunes da Matta » / [A son ami appliqué et à l'éminent élève du Lycée, M. António Ba. Lopes de Oliveira, au nom de l'auteur, ce livre offert par le traducteur, José Nunes da Matta]. 
10 L'explication préalable du «traducteur», qui sert de préface à l'œuvre «d'Henri Montgolfier ", place le traducteur dans la situation similaire de l'étranger : José Nunes da Matta se dit à Guernesey, la nuit du 31 janvier de 1885. Peut-être évoque-t-il la situation des exilés, ou plutôt des expatriés, puisque Guernesey est une île avec une longue histoire de marginalité géographique et politique, recherchée ainsi par ceux qui vivent à la frontière de la vie communautaire : Victor Hugo y a longtemps vécu, et c'est là qu'il a écrit Les Misérables. Cette commune situation d'expatrié, jamais explicite, n'est soutenue que par une douce plainte du traducteur: l'adversité l'a toujours frappé (Montgolfier, 1921: I). En tout cas, Guernesey est un port de passage pour un marin des transatlantiques qui lient l'Europe à l'Amérique ; on y est nulle part et partout.

11 Relevant de la Couronne anglaise, Guernesey est toute proche de la France, de la Normandie; au début $\mathrm{du} \mathrm{xx}^{\mathrm{e}}$ siècle, la langue française y était encore la langue des affaires, tandis que l'anglais était la langue officielle. Sous la responsabilité militaire du Royaume-Uni, elle ne fait pas partie d'un pays, elle ne fait toujours pas partie aujourd'hui de l'Union Européenne... Le coffre, avec le manuscrit d'Henri Montgolfier, tombe du ciel, et José Nunes da Matta en est l'unique témoin. En effet, cette exceptionnalité du témoin est une preuve paradoxale de la vraisemblance : il faut absolument croire au narrateur/ traducteur. Le fait est véridique parce qu'il le dit, puisqu'il déclare la rigoureuse traduction et les circonstances d'un secret absolu. Il doit être véridique puisqu'il justifie l'absence de preuves et nous raconte le vol du coffre et du manuscrit de Montgolfier, unique preuve physique de son histoire. Et finalement, le fait est véridique parce que «le traducteur » le publiera au risque énorme d'être pris pour fou ou pour charlatan (ibid : IIV).

12 Malgré (ou à cause de) la déclaration de fidélité entre les deux textes, celui de l'original et celui de la traduction, «não havendo nela uma única palavra da nossa letra » (ibid: IV); malgré le souci de bien distinguer les notes de l'auteur (signalées par lettres) et les notes du traducteur (numérotées), le lecteur se rend vite compte du contrat tacite de fiction, toujours exprimé en une question de crédibilité. La question de la crédibilité, celle de la présupposition de la vérité absolue, est encore plus importante ici, puisque l'Histoire Véritable de la Planète Mars est le récit «incroyable ", fait par un étranger "méconnu ", d'un voyage « impossible », qui suit les règles du discours utopique, si on veut utiliser la terminologie des genres littéraires.

13 Les trois stratégies de vraisemblance ne peuvent être donc que l'élaboration d'un cercle assez vicieux, où la preuve de l'argument se confond toujours avec l'exposé de l'argument.

- Primo : la méthode tautologique. Si quelqu'un, devant ses déclarations, se met à en douter, il n'a qu'à faire le voyage et rectifier les sources, cela veut dire, aller sur Mars (idem : IV). «L'auteur " / narrateur devient même progressivement plus audacieux quand il parle au « lecteur» / son narrataire. Comment lui décrire l'énorme variété des fruits et des fleurs ? Si le lecteur souhaite y goûter ou seulement les voir, il n'a qu'à chercher la planète, même s'il doit y réfléchir deux ou trois fois avant d'entreprendre le voyage : Henri, « l'auteur », est un survivant aléatoire, invraisemblable, du voyage, « um caso único » (idem : 60). La vérité est au milieu d'un cercle vicieux.

- Secundo : le témoin introuvable. L'identité d'Henri Montgolfier demeure un mystère, même pour le traducteur. Selon Henri, la famille des célèbres Frères Montgolfier n'avait probablement rien à voir avec la sienne, l'auteur du manuscrit (idem : 5). Henri Montgolfier 
montera, symboliquement quand même, dans une montgolfière, protégé par une arche de fer et de liège, et avec ses provisions de vin de Porto, d'eau et de miel...

- Tertio : l'anticipation de l'incrédulité. Les mots sont la matière du rêve, et aussi leur seule évidence. Au début, le doute est si naturel dans la pensée du lecteur que dans la pensée de l'auteur, mais si l'auteur dépasse le doute, s'il témoigne la certitude, alors le lecteur, luiaussi, doit l'accompagner, sans jamais pouvoir mettre en doute l'évidence des faits : « não devo estranhar que haja alguém que duvide, quando eu mesmo chego a recear de que seja um mero sonho (...). Mas não é sonho, não ! É pura realidade, rigorosa e indiscutível » (idem : 20). Au début, la peur que l'auteur éprouve naît, de la possibilité que tout cela s'évanouisse comme de la fumée ou un rêve... Mais si jamais la réalité s'évanouit, s'il en jouit constamment, comment peut-il, le lecteur, en douter (idem : 23) ?

14 À la limite, la vérité n'a besoin d'autres témoins que la déclaration de l'évidence. Même l'intrigue devient une question secondaire et stratégique; le plus important reste toujours l'idée portée, à travers le temps et l'espace, par d'étranges étrangers...

Étranges étrangers tous, et toujours pressés à l'idée de leur mort, toujours guidés par leur responsabilité sociale: le passage d'un témoignage, la perfectibilité de la condition humaine. Montgolfier dédie son œuvre à tous les malheureux habitants de la Terre (idem : 3). José Nunes da Matta publie la traduction même sans l'original, parce que le sujet appartient à tous les peuples de la Terre (idem : IV). Si, après le vol de l'exemplaire, José Nunes da Matta n'arrivera jamais à satisfaire la dernière volonté de l'auteur, en déposant au Musée du Louvre le manuscrit original, il demeurera quand même fidèle à son intention, en publiant la traduction, le seul texte ayant survécu.

Progressivement, le traducteur s'accapare le discours de l'auteur. Au dernier chapitre du livre, le traducteur-auteur (qui éclaire le lecteur sur les fausses similitudes entre la Constitution de la planète Mars et la Révolution des Bolcheviques, en Russie, après 1917) démontre la possibilité d'un tertium genus, une alternative soit à l'intérêt individuel / matériel du système capitaliste, soit à l'intérêt collectif / idéologique du système bolchevique: «Deduz-se, clara e nitidamente, que é possível haver a Moralidade dos Costumes, a Paz entre os Povos, a boa Harmonia e Amizade entre os Homens e uma Felicidade Completa, ao lado da Liberdade, Fraternidade e Igualdade (...) » (ibid : 120). Les idéaux de la bourgeoisie, la Liberté, la Fraternité et l'Égalité, demeurent, selon lui, les idéaux des peuples, même si, entre-temps (c.-à-d. entre le XVIII ${ }^{\mathrm{e}}$ et le $\mathrm{xx}^{\mathrm{e}}$ siècle), la bourgeoisie a oublié les idéaux révolutionnaires, en les interprétant en fonction de l'importance de la Propriété ; et les peuples ont cru aux contrevaleurs de l'oppression, à la haine et à l'égoïsme. Ainsi, ce que ce tertius genus illustre est, vraisemblablement, la possibilité d'une synthèse entre deux discours, qui s'excluent réciproquement: entre « l'utopie » communiste et « l'utopie » capitaliste, l'utopie de Mars est une possibilité ; le meilleur des deux mondes possibles.

Séparés tout avant par le temps et l'espace, Montgolfier et José Nunes da Matta étaient des « étrangers » vis-à-vis de leurs communautés d'accueil (Montgolfier sur Mars, Matta à Guernesey) ; étrangers aussi l'un à l'autre (Montgolfier, un Français du XVIII siècle, Matta, un Portugais du $\mathrm{xx}^{\mathrm{e}}$, ayant besoin de deux langues, d'être traduits). Au début, Matta est un être mélancolique qui se promène seul, la nuit, sur la plage, regardant le ciel, la partie commune de l'univers (idem: I). Même pour les habitants de Mars, cultes et généreux, l'étranger Henri est d'abord un animal, un objet de la curiosité collective. Son corps est placé au Musée Zoologique de la ville de Sarima, et les expériences des savants pour lui rendre la vie ne semblent pas imbues de miséricorde, mais plutôt d'intérêt scientifique. 
Como era de supor, quando por todo o planeta Marte se espalhou a notícia de (...) uma caixa de ferro tendo dentro um corpo morto de um animal parecido com os Martianos, (...) foi geral a anciedade [sic] de ver o corpo inanimado do estrangeiro. Esta ansiedade e comoção subiram de ponto, quando se passou a afirmar de que [sic] os sábios da Universidade de Sarima haviam conseguido insuflar a vida ao corpo inanimado. (idem : 19, c'est nous qui soulignons) ${ }^{1}$ société, tout près des animaux ou des autres êtres animés, même s'il ressemble aux Martiens. Le mot «étranger » est, d'après le texte, non seulement l'identification d'une frontière, mais la frontière même. Le mot sépare, limite. Et ce n'est que peu à peu que le mot devient impropre, inadéquat à l'exercice d'une culture mise en commun. Entre les savants et Montgolfier s'établit alors une notion progressive de similitude, qui se traduit par le programme de convalescence: au début, la chambre de convalescence dans le Musée, mais bien différente des autres qu'il aura l'occasion de visiter (cf. idem: 12s et 16) ; et successivement la promenade à la tour centrale; la fête de présentation à la communauté locale; le voyage à travers les principales villes de la planète; le mariage avec une belle locale; l'intégration dans les services communautaires; et finalement l'écriture de l'Histoire Authentique de la Planète Mars qui, d'après les documents officiels, a pour but la connaissance interplanétaire (idem : 17, 19, 24, 27, 30 et 31).

L'écriture de l'Histoire officielle est, en quelque sorte, le climax de son intégration sociale. De même, tout au long du livre, on constate une identification progressive entre l'auteur (un Français issu de la Révolution française de 1789) et le traducteur (un Portugais issu de la Révolte républicaine, de 1910). Montgolfier commencera à construire son ballon à air chaud en 1804, quelques mois après que Napoléon se fut couronné lui-même. Matta signera la dernière page de son texte le 1er octobre 1921, entre l'ascension du fascisme au parlement italien et les événements de la « Noite sangrenta » au Portugal entre les 19 et 20 octobre (quand un groupe de marins révolutionnaires assassine plusieurs républicains modérés, notamment António Granjo, décédé dans sa cellule à l'Arsenal de la Marine, où il était déjà effroyablement blessé).

Même si on ne trouve pas chez José Nunes Matta une référence explicite à ce contexte politique, comment en faire l'économie ? En quelque sorte, l'écriture de História Autêntica do Planeta Marte (même si elle se présente stratégiquement comme une traduction) s'avère, selon nous, une sorte de vision alternative des événements, et impliquerait une distance critique face aux excès de la révolution républicaine. Le "traducteur » José Nunes da Matta cite l'autorité du Père António Vieira à qui il attribue A Arte de Furtar , pour regretter un pays où quelqu'un est capable de déceler cinquante façons différentes de vol. Mais il regrette davantage encore l'inexactitude de ce nombre, qui arriverait bien à la centaine : « Só os roubos com unhas políticas davam vastíssimo e assombroso assunto (...)» (Montgolfier, 1921: 108). Il suffit de considérer les vols perpétrés par l'avidité politique pour $y$ trouver un thème vaste et surprenant.

José Nunes da Matta est un militaire. Mais le citoyen dont on parle ici méconnaît la brutalité de la guerre et du vol. L'armée de Mars est une institution pédagogique qui surveille et persuade. La société utopique de Mars assimile l'étranger; elle abolit le sentiment de gêne. Les habitants de Mars trouvaient charmantes les erreurs linguistiques d'Henri, ses maladresses d'étranger (cf. idem : 25). Encouragé par un processus naturel d'acculturation, Montgolfier se sentira sur Mars vite "chez lui », séduit par les mêmes paradigmes de beauté, de bonté et de vérité. Il se mariera avec une belle Martienne et sera sensible à la générosité des citoyens et aux arguments des savants. 

mêmes livres, qu'ils partageaient avec les Martiens les mêmes opinions scientifiques, et qu'ils soutenaient les mêmes solutions politiques. Rappelons ici l'explication finale sur les différences ente Martiens et les bolcheviques. Mais aussi les commentaires, aussi bien de Montgolfier que de Matta, sur la valeur de l'hygiène et du confort (idem : 107, 110 et 111), les intuitions de Camille Flammarion sur la sublimité du firmament ou la taille et formation des planètes (idem: $57, c f$. Borralho, 2010: 7), le caractère pédagogique et thérapeutique de la musique et de la peinture (Montgolfier, $1921: 21-23,68$ et 107), mais aussi l'influence morale de l'astronomie, en tant que réflexion sur l'harmonie intarissable de l'univers. En note, le traducteur se réjouit de la similitude entre les opinions des savants de Mars et celles qu'il avait publiées, en solitaire, à Lisbonne, dans l'Annuaire de l'École Navale de 1915 ou dans les Annales du Club Militaire Navale, du deuxième semestre 1921, en ce qui concernait les mouvements de rotation et de translation des satellites d'Uranus et de Neptune, ou les calculs des lignes d'eau des navires à voile et à vapeur ( idem : 35 et 117). On retrouve, sur la planète Mars la sagesse des mesures internationales et même l'adoption de l'Heure Internationale (idem : 57).

Le traducteur constate aussi, à sa grande satisfaction, que ses considérations sur l'importance de l'éducation musicale, si méprisée au Portugal, sont suivies depuis des siècles, sur toute la planète Mars, où l'astronomie est une passion, l'étude du piano est généralisée et la musicothérapie est une médicine triviale (idem : 22s, 68 et 107). Il admire la même gentillesse envers les plus faibles, le même amour pour les arbres (idem: 47 et 60), les enfants, le travail agricole et la discipline scientifique, ou le même soin avec l'hygiène privée et publique (idem: 49, 107, et passim). Les mots de Montgolfier sur les habitudes des Martiens sont mis en relief par les commentaires du traducteur (idem : 107 et passim). Mais aussi par les ouvrages de José Nunes da Matta qui se trouvent toujours aujourd'hui dans nos bibliothèques et qui, quand on les ouvre, nous parlent encore d'un Portugal qui fait abattre les vieux arbres, qui sacrifie la santé publique au faste des monuments, qui publie des lois sans en reformuler l'ensemble de la jurisprudence ou qui méprise la valeur de la littérature et de l'instruction. Lisons, par exemple, A guerra às àrvores feita pela própria lei e a sua nefasta influência na apicultura e turismo (Lisboa, Emp. Nacional de Indústrias Gráficas, 1921), Apicultura prática mobilista (Lisboa, Férin, 1915), A Semana da Criança (Lisboa, Typ. Proença, s.d.), Divagações em verso. Paz e esterilização (Lisboa, Typ. Proença, s.d.), et surtout Instrução literária e sua influência na educação (Lisboa, Typ. Serviços Gráficos do Exército, 1928).

En effet, les villes de Mars sont décrites par le narrateur Henri à l'instar des articles de José Nunes da Matta. Elles témoignent de l'équilibre entre la nature et l'industrie, la science et l'art, la liberté et la sécurité. Le plan d'urbanisation de Mars concilie le confort des maisons familiales avec l'exubérance des jardins botaniques et zoologiques. Le caractère pédagogique des musées, des écoles et des bibliothèques accompagne le pragmatisme des machines électriques, des trains à grande vitesse et des navettes spatiales (plus de $100 \mathrm{~km} / \mathrm{h}$ ). Le spectacle ludique des théâtres semble se confondre avec le spectacle scientifique des observatoires astronomiques... Le spectacle s'avère partout l'exercice de l'observation méthodique (Montgolfier, 1921: 24). L'utopie est une méthodologie. À l'instar des utopies sociales de More ou de la fin du XIX vision de la cité idéale est un modèle généreux pour la propriété ; un modèle à suivre par tout le monde, acquis à l'évidence du bonheur collectif (cf. Mucchielli, 1982: 55-103). Le désir prouve d'ailleurs la possibilité de l'utopie: «A quem fizer a leitura desta singela 
descripção de Montgolfier não é para admirar que lhe venha à ideia a tentação frenética de fugir da Terra e de ir viver para Marte »(Montgolfier, $1921: 108)^{2}$.

La Terre n'est pas Mars, il est vrai : Montgolfier pleure quand il est heureux, bégaye quand il tombe amoureux (idem: 25-30 ss). Par contre, les Martiens, qui prônent le naturel de l'activité sexuelle, se méfient de l'amour. Ils conçoivent le mariage comme un contrat d'eugénisme ou un épanouissement physique socialement contrôlé, et réduisent au minimum les contacts sociaux puisque les baisers et les accolades propagent les maladies (idem : 87-94, et 101). Pour ce qui est des questions de l'origine des espèces, la théorie dominante chez les savants de Mars est celle du croisement progressif des plusieurs espèces de primates, tandis que José Nunes da Matta défend, de son côté, que l'origine de l'homme est directe (idem : 62).

Mais la Terre est déjà Mars, sauf pour ces rares exceptions. L'identification entre les trois narrateurs (Montgolfier, Matta, et Constantinio, le réformateur du peuple de Mars) semble presque parfaite. Si parfaite qu'elle transforme en effet l'utopie (un espace sans lieu) en une uchronie (une époque sans temps). L'uniformité de l'espace physique décrit et admiré, l'uniformisation de la langue, l'hybridisme des races forcé par les contrats de mariage interraciales, la similitude des maisons, l'absence de propriétés agricoles privées et la discrétion de tout signe extérieur de richesse, la permanence immémoriale du régime démocratique, la paix universelle instaurée, le caractère cyclique du climat ou le curieux système de recyclage de l'industrie, contribuent certainement à cette incapacité de mesurer le temps, propre de l'état uchronique.

L'utopie ne fait que ralentir la furie du temps réel qui nous charrie. Les mesures du temps utilisées sur les deux planètes contraignent à un rythme plus lent sur Mars et plus accéléré sur Terre : le jour sur Mars est plus long de 34 minutes, moins 29 secondes, et l'année tropique de Mars correspond grosso modo à 687 de nos jours terrestres (idem : 55). Certes, ce temps psychologique plus lent ralentit les saisons, le changement de l'espace et l'avidité des habitants de Mars. Mais il faut surtout souligner l'importance d'un état léthargique qui nous empêchera toujours de faire la correspondance entre le temps de la Terre et celui de Mars.

Entre le départ vers l'Espace, en 1804, et la publication du livre à Lisbonne, en 1921, Montgolfier subit le temps indéfini et relatif du voyage, transformé par la vitesse de son bolide. Et ce temps du voyage finit par être, surtout pour son protagoniste, un temps sans mesure, le temps de la mort. Entre la Terre réelle et la Terre Promise (Mars), la mort symbolique s'impose. C'est elle qui annonce la naissance au nouveau monde. Montgolfier se dit alors " ressuscité ", incapable de dire si son état léthargique a duré quelques années ou quelques siècles (Montgolfier, 1921 : 11). À la fin, il envoie 50 exemplaires de son récit sur Terre, mais il se déclare incapable de calculer la durée de ce voyage. Et cette indéfinition du temps, encore plus que celle de l'espace, nous fait perdre conscience des limites de cet espace. À la limite, elle détruit même la notion d'« étranger " puisque Montgolfier naît une deuxième fois, à la planète Mars et, tout comme José Nunes da Matta, le traducteur, nous nous identifions à lui, grâce à la $1^{\text {ère }}$ personne et aux autres stratégies d'empathie à l'œuvre dans leurs discours.

Cette uchronie vécue par l'étranger qui progressivement laisse de l'être est un est un des pièges de l'utopie: si quelqu'un, où que ce soit, est capable d'imaginer un monde plus parfait, si quelqu'un a été capable de reproduire ce monde à une échelle individuelle, alors l'utopie est possible, ne dépendant que de la volonté d'un plus grand nombre d'individus, toujours guidés par le même désir. Rien ou personne n'empêche vraiment 
l'utopie. Comme dans le livre de More, même la civilisation technique n'est pas une cause de l'utopie; elle en est une des conséquences. Pour le reste, la volonté individuelle et politique suffisent. Les quatre lois majeures de la constitution de Mars font l'éloge du mélange linguistique, social, racial, culturel ou économique: l'adoption d'une langue commune, la centralisation d'un pouvoir démocratique, le programme de fusion des races et le contrôle de la population (voir les arguments pour et contre, Montgolfier, 1921: 74-87).

Mars est déjà la Terre. L'utopie ne fait qu'augmenter le détail que nous avons perdu de vue. Autrefois, il y a cent mille ans, Mars a également connu la guerre, la peste, la lutte pour la possession du pétrole ou du charbon, presque épuisés. Ses habitants se battaient entre eux ayant pour prétexte la survie des nations et des races. L'égoïsme et l'avidité des richesses individuelles ont presque détruit les ressources naturelles de la planète, provoqué l'abandon des champs agricoles, diminué la diversité de la faune, de la flore. Le droit, la science et même les arts et la littérature, ont été mis au service de la force des plus forts contre la faiblesse des plus faibles ((idem : 42, 68-72 et passim). Ils ne légitiment que la violence qui, à la limite, se passe toujours de légitimité. La qualité de vie est devenue insupportable pour une large partie de la population. La Guerre et la Peste, violences radicales des Hommes et de la Nature, ont provoqué in extremis une mort symbolique, cette fois-ci collective, décrite parfois avec les mêmes sentiments d'incrédulité et de déchéance. La population de Mars subit donc, comme l'étranger, tout d'abord un état léthargique et, ensuite un état de résurrection, - la Révolution Sociale ( $c$. idem : 72 ss.) -, peut-être issu d'un stock de vitalité similaire («stock de vitalidade ») que possèdent les animaux, et dont nous parle Montgolfier (cf. idem : 14).

La Terre est encore Mars. Autrefois, il y a des milliers d'années, la destruction d'une des planètes de notre système solaire aurait déplacé l'axe de rotation de Mars. La turbulence aurait aussi déclenché plusieurs cataclysmes sur la surface de la Terre. Le lac de la Méditerranée s'est ouvert et une des sociétés les plus civilisées de la Terre a été engloutie par les eaux de l'Atlantique. Le traducteur compare alors l'information des Martiens, révélée par Montgolfier, avec les informations de Platon, conservées dans Critias et Timaeus. Il rappelle les légendes sur l'existence de ce royaume aux Açores ou aux Canaries, et le mythe biblique du Déluge ou les actions violentes de Mars, dieu de la mythologie latine. Matta nous parle encore des récits sur l'Atlantide (idem : 37-39 et 56) et se réjouit à nouveau de la similitude des opinions des Martiens et les siennes, inclues dans son livre $O$ Passado (idem : $38 \mathrm{~s}$ ), plus précisément, nous semble-t-il, Rápido golpe de vista do Passado, Presente e Futuro, un livre que Matta publiera onze ans plus tard (s.l., s.n., 1932).

En effet, nous croyons trouver dans ce récit plusieurs axes de correspondance qui se légitiment mutuellement. On y trouve une même exemplarité, de plus en plus évidente, qui va des possibilités de l'individu à celles des communautés, qui nous conduit des réformes d'une communauté imaginée à celles d'une communauté réelle; qui nous propose l'étrange vision des étrangers (Montgolfier et les Martiens) comme un principe sine qua non du cosmopolitisme scientifique ou social (de José Matta). Peut-être est-ce l'efficacité de cette structure de macro ou microcosme, en matriochka, qui séduit le lecteur.

Mais peut-être l'importance de la perspective de l'étranger s'éclaire-t-elle aussi par la réception littéraire du récit de José Nunes da Matta. Le Galicien/Brésilien Modesto Brocos (qui, en 1930, publie son Viaje a Marte, à Valencia, Arte y Letras) a sans doute été séduit par le texte de Matta et par cette figure de l'étranger interplanétaire. L'œuvre de 
Brocos, étant extrêmement rare et invariablement ignorée par les études utopiques, même en Espagne, nous avons longtemps supposé que Viaje a Marte était une œuvre originale. C'est seulement un article d'Agustin Jaureguízar, publié en 2009 dans la revue Arbor, qui nous a permit l'identification de cette adaptation livre de l'História Autêntica do Planeta Marte.

Quelques détails biographiques sont certainement utiles. Modesto Brocos (né à Saint Jacques de Compostelle en 1852 et décédé à Rio de Janeiro en 1836) est contemporain de José Nunes da Matta (né en 1849 et décédé en 1945 à Lisbonne). On ne peut pas garantir quand et comment Brocos a connu le récit de José Nunes da Matta. Brocos est un peintre académique, professeur et auteur d'une "Rhétorique des Peintres ». Après avoir étudié à Buenos Aires, à Paris, à Rome, à La Corogne, il se fixe définitivement au Brésil en 1890, et c'est au Brésil qu'il devient professeur de l'École Nationale des Beaux-arts et un peintre assez connu (il est l'auteur de la plupart des tableaux qui ornent la vieille Bibliothèque Nationale de Rio de Janeiro). On lui connaît quelques ouvrages en portugais. Entre 1879 et 1881, il collabore à la revue Illustración Gallega y Asturiana dirigée par l'historien Manuel Murguía (marié avec Rosalía de Castro) et constructeur, entre autres, d'une nouvelle conscience de l'identité de la Galice. Viaje a Marte, le récit de Brocos, est le seul ouvrage de fiction de l'auteur et a la particularité d'avoir été écrit en espagnol (1928) et publié à Valencia (1930), alors que Brocos était âgé de 78 ans.

On peut bien sûr la situer dans le contexte littéraire espagnol (cf. Álvarez de Miranda, 1980) et la considérer héritière des voyages sur la lune du XviII ${ }^{e}$ siècle de Diego Torres de Villaroel (1724), du Discours IV de El Observador de l'Abbé José Marchena (1787), d'António Marqués y Espejo, auteur de Viaje de un filósofo a Selenópolis, corte desconocida de los habitantes de la Tierra (1804), qui est en fait une traduction de Le voyageur philosophe dans un pays inconnu aux habitants de la Terre (1761) de Daniel Villeneuve. L'invention des ballons à air chaud a multiplié les récits utopiques, plus ou moins sublimés, plus ou moins enflammés pour des raisons amoureuses (cf. Lynn, 2010). La tendance s'inscrit sans doute dans un contexte plus vaste encore, celui de la Littérature Occidentale, qui commence avec l'Histoire Véritable de Lucien de Samosate, clairement évoquée d'ailleurs par le titre de l'ouvrage de José N. Matta, et qui passe forcément par les récits de Kepler, Francis Godwin, John Wilkins ou de Cyrano de Bergerac au XVII siècle; Fontenelle, Robert Poltock, Voltaire au XVIII ${ }^{\mathrm{e}}(c f$. (Hatzenberger, 2010), Poe ou Verne au XIX ( $c f$. Horowitz, 1986 : passim). Mars est une planète qui connaît une certaine vogue à partir de la fin du XIX ${ }^{e}$ siècle. Les récits de Gustavus W. Pope et d'Edgar Rice Burroughs (repris en 2011 par le film John Carter), ou même The war of the words, d'H. G. Wells, oscillent entre l'utopie et la dystopie, puisque Mars est encore la planète du dieu de la Guerre. Jaureguizar en cherche les influences possibles.

Brocos demuestra que ha leído algo sobre él porque hace suya la teoría de los canales marcianos - y susceptible de haber conocido una antigua civilización. Quizá también por la notoriedad y el éxito alcanzados por la película soviética Aelita, una utopía socialista en Marte rodada en 1924 por Yakov Protazanov sobre la novela del mismo título de Alexis Tolstoi. (Jaureguizar, 2009 : 1314)

C'est possible. La mode des voyages interplanétaires et les utopies portugaises et espagnoles font l'objet de redécouverte dans les archives (Reis, 2007, Borralho, 2004, cf. Ameal, 1979, Alvarez de Miranda, 1981). Les dialogues interdiscursifs sont nombreux. Mais le rapport qui s'établit entre les textes de José Nunes da Matta et Modesto Brocos est d'une plus grande proximité. Le texte de Brocos n'est pas une traduction fidèle du livre de 
José Nunes da Matta, publié en 1921, mais il garde la plupart de sa structure et des détails. Faisant l'éloge de la liberté individuelle et de l'absence de propriété privée, elle est considérée par Augustín Jaureguizar un bon exemple des utopies socialistes. Les deux auteurs choisissent Mars pour espace édénique; stratégie assez rare, puisque le nom évoque le dieu de la violence. Brocos, au Brésil, évoque d'une façon plus nostalgique, les champs verts de son pays natal, ceux de Pontevedra, mais le paysage qu'il décrit est encore celui de José Nunes Matta, une sorte de campagne en ville. Plus spécifique encore est le nom de l'habitant de Mars qui montre à Brocos les merveilles de la planète. Il s'agit de Fray Benito Feijoo, le Galicien du XVIII ${ }^{\mathrm{e}}$ siècle, auteur du Teatro Critico. Pourtant, dans les deux cas, les descriptions et le discours didactique, surtout dans le texte de Brocos, éliminent presque l'intrigue. Si José Nunes da Matta est le traducteur d'un texte de Fénelon, souvent associé à la tradition utopique (A sabedoria humana ou o Retrato de um homem honesto, por Fénélon, Parede, Typ. Proença, 1942), c'est encore la lecture de Fénelon qui revient à l'esprit de Jaureguizar quand il lit Brocos, même si Jaureguizar ne connaît pas l'ouvrage de Matta :

Hay que decir también que es una obra del más completo estatismo narrativo en aras del adoctrinamiento políticoreligioso. Sigue en buena medida la forma - plena de diálogos y, aún más, de monólogos interminables - del Telémaco de Fenelon, con un Feijóo que juega el rol de Mentor y un Brocos que desempeña el papel de Telémaco. (Jaureguizar, $2009: 1316$ )

La lecture de Matta par Brocas est indéniable. On retrouve à présent les idées de Constantínio dans la bouche de Feijoo: le programme méticuleuse d'eugénisme, l'union des races à travers le mariage, l'unité linguistique et politique de toute la planète, l'efficacité de la justice, l'égalité des sexes, l'élimination des entreprises de distribution et l'éloge du mouvement coopératif, le caractère pédagogique de l'armée, la garantie de la santé et de l'éducation publiques...

38 Le texte de Brocos ne s'avère plus incisif que quand il parle de la naturalité du désir sexuel... Si José Nunes da Matta décrit une société où le mariage contrôle ce désir en contenant les excès et en ne privant personne de sexe, même s'il faut admettre la polygamie (masculine), Modesto Brocos réunit les femmes non-mariées dans une sorte de monastères, les maisons de "las Hermanas Humanitarias ", leurs services soutenant les causes humanitaires...

Peut-être le texte de Brocos est-il en général un peu plus invraisemblable. Dès les premières pages, Brocos fait de Brocos le personnage qui arrivera sur la planète Mars et l'auteur du texte. Le lecteur se demande bien comment il peut lire son livre sur Terre. Même choc du lecteur pour la conclusion de ce contrat de crédibilité : Brocos est en train de visiter un musée quand il écrit (ou nous décrit) : «me acometió un vértigo, perdí el equilibrio y...» (apud Jaureguizar, 2009: 1321). Comment comprendre les points de suspension? Signifient-elles la mort subite du personnage, la fin d'un rêve? Mais pourquoi la mort, dans cet univers littéraire qui permet de tout « lire »? Et où commence le rêve?

Les rapports établis entre les textes de José Nunes de Matta, Modesto Brocos et leur contexte littéraire et politique rendent pertinente une plus ample réflexion sur la fonction de l'étranger et la morphologie du voyage (cf. Vogler, 2006). Ce n'est pas par hasard que les caractéristiques des étrangers qui peuplent ces planètes ressemblent énormément à celles des contes qui ont pour protagonistes les héros "Sans Peur ". Greimas a élaboré un magnifique ensemble de considérations sur les fonctions atypiques 
qu'ils opèrent dans le récit (Greimas, $1970: 231$ ss.). L'étranger, comme un héros « sanspeur ", se caractérise par son attitude provocatrice. Il s'enfuit quand les autres acceptent de rester; il répond quand les autres se limitent au silence ; il cherche la liberté quand la communauté choisit la sécurité... Sa condition d'étranger est le plus souvent la conséquence du courage de sa fuite vers un espace qui est (pré)visiblement inhospitalier.

Pourtant, son « ouverture » (Propp, 1983 : 66) nous semble régulièrement suivie par cinq fonctions qui nous rappellent celles du philosophe dans le mythe de la caverne de Platon (dans la République, Livre VII) ou du pèlerin du texte de Parménide (Sur la Nature) :

a. L'étranger atteint une condition extrême de dépouillement ou d'indifférence. Volontairement, comme dans l'Hytlodée de More, ou involontairement, comme les marins qui pris dans une violente tempête, dans le récit de Lucien de Samosate, le protagoniste perd ce que les autres ont de désirable. Dépouillé, il n'est plus qu'un homme.

b. Les éléments de la nature ou la curiosité démesurée entraînent le protagoniste dans un espace inconnu, presque inaccessible, impossible à atteindre à la limite. Le passage est toujours une épreuve physique. Cyrano confond son enthousiasme avec une maladie; Hans Pfaall, chez Poe, est au bord du suicide. Les éléments de la nature (le feu d'un bolide ou de la fièvre, l'eau de la mer ou d'une boisson, l'air raréfié de l'atmosphère ou la terre d'une grotte profonde) menacent sa vie. Il devient un corps inanimé.

c. Arrivé sur une autre planète, au bout du monde, le protagoniste se rend compte de sa nouvelle condition d'étranger. Ce fait cautionne simultanément les préjugés du lecteur (qui n'a jamais quitté la Terre) et assure les hardiesses des habitants du lieu. Par le biais d'un étudiant de Tycho Bahe endormi, Kepler peut défendre ouvertement le système copernicien. Ce qui est vérité et coutume pour les uns devient mensonge et bizarrerie pour les autres. Mais le résultat est, au moins, une relativisation de la vérité et du mensonge. L'étranger est le pont que traversent les uns et les autres.

d. Détenteur d'une vérité nouvelle, d'une sagesse qui advient de la synthèse des extrêmes ou de l'enthousiasme pour l'Éden terrestre, cet étranger devient véritablement un habitant de cet Éden. Et c'est inévitablement quand il se rend compte qu'il n'est plus un étranger qu'il se souvient de sa première patrie, celle où il avait été malheureux, celle qu'il avait quittée sans regret. Il sait, de surcroît, qu'il n'y sera jamais bien compris. Les protagonistes de Un homme sur la Lune de Poe sont pris pour des charlatans, des ivrognes ou des fous. Comme le philosophe ou le pèlerin, l'étranger reviendra un jour. Ou bien il écrit ses mémoires, pour passer son témoignage. Le narrataire devient narrateur.

e. Ainsi, une dernière fonction lui est-t-elle réservée: celle d'assurer l'instruction de la postérité. L'étranger parle souvent d'un monde futur qui sera finalement capable de comprendre ce qu'il dit, et l'urgence de ce qu'il dit. Peut-être ce qui est maintenant impossible deviendra-t-il un jour inévitable. L'étranger revient. Et il est encore un étranger. Cette fois-ci pour les habitants de son pays de départ. Toujours dépaysé, il évoque alors le Temps, il le contrôle. À travers l'Histoire, à travers le Mythe, à travers la Littérature... Il devient agent provocateur.

42 La fin de l'Utopie, c'est l'Uchronie, cet «à travers...» successif, ces points de suspensions..., cette ambiguïté d'une humanité qui recommence à chaque fois par le premier cri d'un nouveau-né, cette répétition entêtée. Du moins pour cet éternel étranger, ce juif errant qui nous gêne. 


\section{BIBLIOGRAPHIE}

ÁlVAREZ De MIRANDA, Pedro (1981). « Sobre utopías y viajes imaginarios en el siglo XVIII español », Homenaje a Gonzalo Torrente Ballester, Salamanca : Caja de Ahorros, pp. 351-338.

AMEAL, João (1979). « Não há Utopias Portuguesas », Sep. de Revista de História da Sociedade e da Cultura da Universidade de Coimbra, Coimbra, FLUC/ CHSC, pp. 163-170.

BorRalho, Maria Luísa Malato (2004). « Não há Utopias Portuguesas? », Estilhaços de Sonhos : Espaços de Utopia, Porto : Quasi, pp. 58-73

BORRALHO, Maria Luísa Malato (2010). « Sobre nós uma abóbada estrelada. Breves citações de astronomia na Literatura » [On-line], in E-Fabulations, n.. 7, Porto, pp. 7-20 [disponible le 9/2/2012]. <http://ler.letras.up.pt/uploads/ficheiros/8663.pdf>

Brocos, Modesto (1930). Viaje a Marte. Valencia : Arte y Letras.

Greimas, Algirdas Julien (1970). Du Sens. Essais Sémiotiques. Paris : Seuil.

HATZENBERGER, Antoine (ed.) (2010). Utopies des Lumières. Lyon: ENS.

HoRowitZ, N. H. (1986). To Utopia and Back: The search for life... New York: W. H. Freeman.

JankeleVitch, V. (1979). L'ironie, ou la bonne conscience. Paris: Flammarion.

Jaureguizar, Augustin (2009). «El Viaje a Marte de Modesto Brocos » [on-line], in Arbor. Ciencia, Pensamiento y Cultura, no CLXXXV 740, nov.-dic. pp. 1313-1322 [disponible le 9/02/2012]. <http:// arbor.revistas.csic.es/index.php/arbor/article/view/397>

LimA, J. A. Pires de (2010). Vice-Almirante José Nunes da Matta. Breve biografia. s.l.: ed. Autor LYNN, M. R. (2010), The Sublime Invention. Ballooning in Europe. London: Pickering \& Chatto. MANNHEIM, Karl (1960). Ideology and Utopia. London : Routledge \& Kegan Paul.

MARQUÉS Y ESPEjO, A. (2007). « Viaje de un filósofo a Selenópolis » [on-line], ed. J. C. Martínez García. s.l. : Paiperez [ disponible le 9/2/2012]. <http://www.liceus.com/cgi-bin/ac/pu/el\% 20viage\%20de\%20un\%20filosofo\%20a\%20selenopolis\%20por\%20D.A.M.E.pdf>

MATTA, José Nunes da (1928). Instrução literária e sua influência na educação, [s.n.] : SGE.

Montgolfier, Henri (1921). História Autêntica do Planeta Marte, trad. José Nunes da Matta. Lisboa : Typ. Cooperativa Militar.

MoRUS, Thomas (2006). Utopia, ed. Aires A. Nascimento. Lisboa : F. C. Gulbenkian.

MucCHIELLI, R. (1982). Le Mythe de la Cité Idéale. Brionne : G. Monfort.

NEussüs, Arnhelm , ed. (1971). Utopía, trad. Maria Nolla, Barcelona : Barral.

Propp, V. (1983). Morfologia do Conto, trad. J. Ferreira e V. Oliveira. Lisboa: Vega.

REIS, José Eduardo (2007). Do espírito da Utopia : Lugares utópicos e eutópicos, tempos proféticos nas culturas literárias portuguesa e inglesa. Lisboa : Fundação Calouste Gulbenkian.

Trousson, Raymond (1979).Voyages aux Pays de Nulle Part. Bruxelles : Ed. de l'Université. 
VOGLER, Christopher (2006). A jornada do escritor: estruturas míticas para escritores, trad. Ana Maria Machado (The Writer's Journey), 2. ed, Rio de Janeiro: Nova Fronteira.

\section{NOTES}

1. «Comme il fallait s'y attendre, quand la nouvelle de la chute (...) d'une boite en fer contenant le corps mort d'un animal semblable aux Martiens s'est propagée sur toute la planète Mars, l'envie de voir le corps inanimé de l'étranger fut générale. Cette hâte et cet émoi atteignirent leur apogée quand on en est arrivé à affirmer que les savants de l'Université de Sarima avaient réussi à insuffler la vie dans le corps inanimé ».

2. Celui qui fera la lecture de cette singulière description de Montgolfier sera certainement tenté de fuir frénétiquement la Terre et d'aller vivre sur Mars).

\section{RÉSUMÉS}

La définition d'« étranger " est indéniablement liée à la définition d'« espace » : l'étranger est celui qui est étrange à un espace réel, un espace individuel ou national, notre espace, politique et/ ou physique, tout d'abord. Mais l'étranger est aussi celui qui est étrange à notre espace imaginaire, mythique et symbolique, celle-ci étant peut-être la dimension la plus importante de l'espace réel. C'est pourquoi nous avons trouvé significatif un livre qui se base sur les équivoques entre les espaces réels et imaginaires: La Véritable Histoire de la Planète Mars, faussement attribuée à Henri Montgolfier, voyageur français. La description de Mars, écrite in loco selon l'éditeur, serait traduite et publiée par un Portugais, José Nunes da Matta, lui aussi séduit par les voyages à travers l'espace (et adapté par Modesto Brocos, un peintre galicien). Ils nous obligent à mêler ainsi les espaces politiques, physiques, imaginaires et symboliques (ceux de la France, du Portugal et de Mars), à repenser la définition «d'étranger » et à concevoir ses fonctions dans le récit utopique.

The definition of "foreigner" is unavoidably connected with the definition of «space ». The foreigner is a person who is, first of all, «strange » to a political space, to a physical space, our space. But he is also, or specifically, the one who is strange to an imaginary, mythical and symbolic space that is called « individual» or « national culture ». For those reasons, it seems to us important to talk about a peculiar and rare book, published in Lisbon, in the year of 1921: The Truly Story of the Planet Mars, allegedly attributed to a French traveler, Henri Montgolfier. The description of the planet, written "in loco ", would be finally translated by a Portuguese, José Nunes da Matta, also seduced by space travels (and adapted by Modesto Brocos, a painter born in Galicia, which impels us to cross also political, physical, imaginary and symbolic spaces, in France, Portugal and Mars, to redefine «foreigner ", and to considerer its functions in utopia.

\section{INDEX}

Mots-clés : étranger, voyage, utopie, Mars, Nunes da Mata (José), Brocos (Modesto)

Keywords : foreigner, travel, Utopia, Mars, Nunes da Mata (José), Brocos (Modesto) 


\section{AUTEUR}

MARIA LUÍSA MALATO BORRALHO

Universidade do Porto

mlmalato[at]gmail.com 